\title{
Planning, Control and Monitoring Tools for Industrial Garment Assembly Processes
}

\author{
Helder Carvalho \\ University of Minho \\ Dep.Textile Engineering \\ Campus de Azurém \\ 4800-058 Guimarães, \\ Portugal \\ helder@det.uminho.pt
}

\author{
Ana Rocha \\ University of Minho \\ Dep.Textile Engineering \\ Campus de Azurém \\ 4800-058 Guimarães, \\ Portugal \\ amrocha@det.uminho.pt
}

\author{
João L. Monteiro \\ University of Minho \\ Dep.Industrial Electronics \\ Campus de Azurém \\ 4800-058 Guimarães, \\ Portugal \\ monteiro@dei.uminho.pt
}

\author{
Luís F.Silva \\ University of Minho \\ Dep.Mechanical Engineering \\ Campus de Azurém \\ 4800-058 Guimarães, \\ Portugal \\ lffsilva@dem.uminho.pt
}

\begin{abstract}
Garment construction is a complex process due to the properties of the textile materials. Furthermore it still relies on human operators in most of the material handling and process monitoring. On the other hand, the set-up of the machines is still based on trial-and-error and empirical knowledge. Current market trends with higher demand on quality, shorter production runs and increasing variety of materials boost the economical significance of machine set-up and better process planning, monitoring and control.
\end{abstract}

This paper presents the development of monitoring and control techniques aiming at the introduction of new tools to accomplish this. A sensor-based data acquisition and analysis system has been developed that enables a quick offline evaluation of the performance of the machine in several of its subsystems. Knowledge that has been gained from these measurements is the basis to develop $\mathrm{CAE} / \mathrm{CAM}$ tools and real-time control devices.

\section{Introduction}

The industrial production of garments and similar textile end-products is an activity that has roots back to the $19^{\text {th }}$ century, and that has profited immensely from continuous technological developments since its first emergence. These developments reflect on important aspects of the industrial production infrastructure, as are product design and development, production planning and management, and the manufacturing equipment itself. But several characteristics of the materials that are processed give rise to technical issues that are difficult to objectively manage and control.

The first of the characteristics of textile materials significant in this aspect are its limpness and elasticity. These properties make automated material handling and transportation within the shopfloor, and at the individual assembly operations, very difficult to automate. Although equipment manufacturers have continuously been developing all kinds of auxiliary devices aimed at increasing productivity and quality, it can be observed that almost all of the operations involved in garment assembly still depend on the human operator or on its supervision (especially in sewing operations).

The second important aspect is the very unpredictable behaviour of the materials when they undergo the manufacturing operations. Garment assembly involves fabric spreading and cutting, part assembly, most frequently by sewing them, and ironing/forming operations. Each of these types of operation poses its own problems related to the nature of textile materials. The upfront engineering of the process in all of the mentioned operations has been studied along the years by Textile Science, and many advances have been made.

In the sewing operations, however, most of process design is still based on subjective and empirical judgement. The set-up and configuration of the machines is determined by producing samples and setting the machines on basis of trial-and-error that is a time consuming operation and relies on the operator's experience. The machines, although often equipped with complex devices to aid in material handling, are blind regarding the process itself; they run with an initial set-up and are neither capable of adapting to varying sewing conditions nor can they detect anomalous operation. This means that both the correct setting of the machine as well as control of the process and supervision of quality rely entirely on the skills of human operators.

To achieve the objective of a more complete and, in particular, more objective control, the first step is a better understanding of the process and of the interactions of machine settings and configurations, objective material properties, and process efficiency/product quality. This is one of the motivations for several researchers and manufacturers to perform studies using sewing machines devised with sensors to measure several process variables in real-time[1][2][3][4]. Another motivation is the increasing need to quantitatively monitor and even document sewing conditions concerning high-tech seams (air bags, safety belts, technical textiles), in the framework of quality certification. 


\section{Sewing process variables and its measurement}

Industrial sewing machines are available in an enormous variety. They may be classified by their stitch type, speed, application, or degree of automation. The fastest machines can reach sewing speeds of up to 10000 stitches per minute (spm), being normally applied in fully automatic sewing systems. Only the simplest operations may be completely automated. More complex operations are always performed by human operators, on machines equipped with a range of auxiliary devices, depending on the specific sewing operation.

In all of these machines the basic mechanisms for seam formation are similar. To accomplish the production of a seam, the following functions are necessary:

\section{a) Crossing the fabric with a thread}

This function is performed by the needle that is inserted in the needle-bar (Fig.1). To measure needle penetration forces, a piezoelectric sensor has been introduced. The sensor is subject to motional forces, thread forces and to penetration forces, but only the latter are of interest to the measurement. Thread forces are eliminated by unthreading the machine. This procedure is naturally only possible for laboratorial studies. Motional forces are eliminated by specifically designed filtering procedures. These unavoidably introduce an error to the measurement, which can thus only be considered as an estimate, especially when working with thread [5].

Measurement of needle penetration forces is important to aid in needle choice and/or to pinpoint fabrics potentially causing sewing problems. Ideally, penetration should be monitored in real-time in order to detect sewing defects caused by the needle in-process.

b) Interlacing thread(s).

The stitch type produced determines how this is specifically achieved. Stitch formation occurs by the synchronised movement of the needle, feeding system and other stitch formation elements.

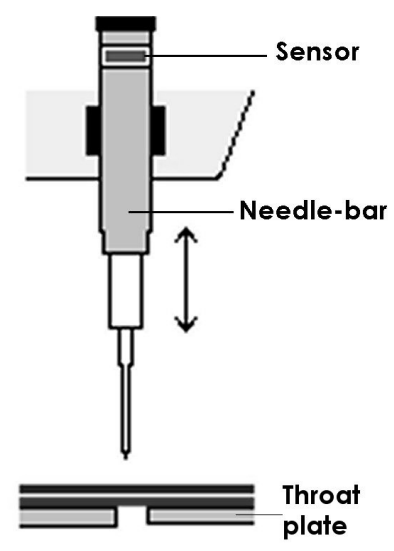

Fig.1: Needle-bar force measurement
In the case under study, two loopers are used, each of them carrying a thread. The stitch formed by the machine is classified as type 504. It is represented in Fig.2.

To study the stitch formation process, the machine has been equipped with strain-gauge based sensors to measure the dynamic forces produced on the threads during stitch formation. A complementary measurement is that of average thread consumptions, that is achieved by three rotary encoders and a specifically developed microcontroller-based device. The quantitative description of stitch formation can be used to both detect localised defects in stitch formation, as well as to provide feedback for automatic thread tension control.

An additional development that is currently being undertaken is a measurement related to static thread tension. Tension is introduced into the threads by tensioners, which consist of two concentric metallic disks compressed by a spring. The thread passes between these disks and acquires tension according to the adjusted compression force of the spring. The introduction of a low-cost sensor to measure this compression force is expected to be an important tool to ease controlled thread tension adjustment.

c) Feeding the material (moving it forward a predetermined distance - the stitch length)

This is the third function for the production of a seam. The simple drop-feed mechanism, represented in Fig.3, uses a feed-dog underneath a throat plate.

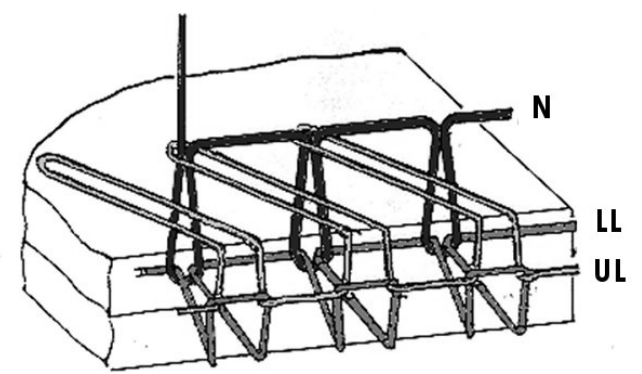

Fig.2: Three-thread overlock stitch type 504 (N: Needle thread, LL: Lower Looper thread, UL: Upper Looper Thread)

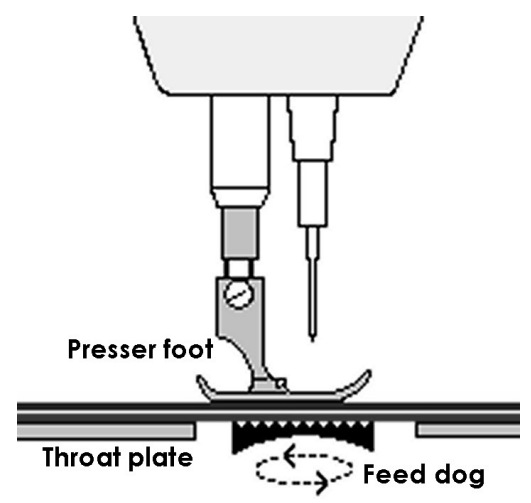

Fig.3: Basic feeding mechanism 
The throat plate has slots through which the feed-dog can pass, in its elliptic movement, to press the fabric against the presser-foot and forward, leading the seam to acquire length. The presser-foot is traditionally devised with a spring set to a determined pre-tension. The evaluation of feeding performance is achieved with a piezoelectric force sensor inserted coaxially with the spring, and with an LVDT measuring vertical displacement.

Seam defects may be produced in any of these three functions and may cause the workpiece to be rejected by either aesthetical or functional criteria. In the latter case, seam strength or reliability is an issue that may be a determining factor in special applications.

\section{The set-up of a test rig}

Fig.4 shows a general overview of the sewing test rig developed. The sensors placed on the overlock machine are plugged into custom conditioning hardware that connects to a data acquisition board installed in a PC.

A software application developed with Labview provides all the functions necessary for signal acquisition, processing, display and storage, sensor calibration and hardware control.

Most importantly, the software includes a generous set of tools to analyse the acquired signals. These started with general signal analysis tools (peak detection, spectral analysis) but in the course of the project additional modules have been developed specifically to characterise the state of the sewing process in all three subsystems.

Fig.5 presents a screenshot of these sewing efficiency test modules showing some parameters relevant to evaluate presser-foot trajectory, namely peak displacement values, signal spectrum and harmonic distortion. For the displacement signal, 2 peaks and a valley of the signal are detected within certain phases of the stitch cycle.

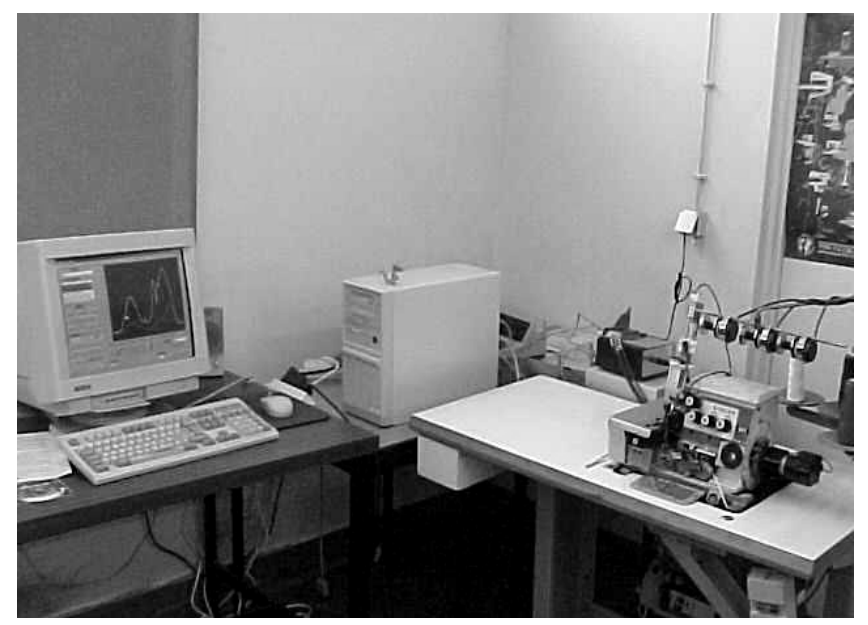

Fig.4: Overview of the sewing test rig
One possible representation is that of Fig.5, in which each dot represents a stitch, with its coordinates being the peaks and valleys computed for it.

Based on these software tools, several experimentation series were run to test stitch formation, feeding efficiency and needle penetration in varying situations[6][7][8]. The parameters provided by the test tools were observed in situations of good and bad sewing performance. This work has led to a set of very interesting results. Although it is still early for the development of fully automatic control for all parameters - that could be envisaged as a mediumterm objective of this research - the development of interesting applications is possible and is being undertaken by the team.

\section{Results}

\subsection{Feeding system}

The results obtained with presser-foot displacement measurement are notable. The information provided by this measurement clearly depicts the operation of the feeding system. Losses of contact between fabric and presser-foot, a cause for stitch length irregularity, can be detected by various methods. The definition of Admissible Displacement Limits (ADL)[6] with which the peak values are compared, is one of them. An alternative is the comparison of the measured displacement cycles with a reference cycle that is obtained at low speed (Fig.6). Sewing defects like folds or curling of the fabric at the edge are readily detectable by the ADL method or by monitoring the difference of displacement to its reference. The extraordinary sensitivity of the measurement allows the detection of even very fine differences in sewing conditions, such as the amount of stretch that is given to the fabric during sewing.

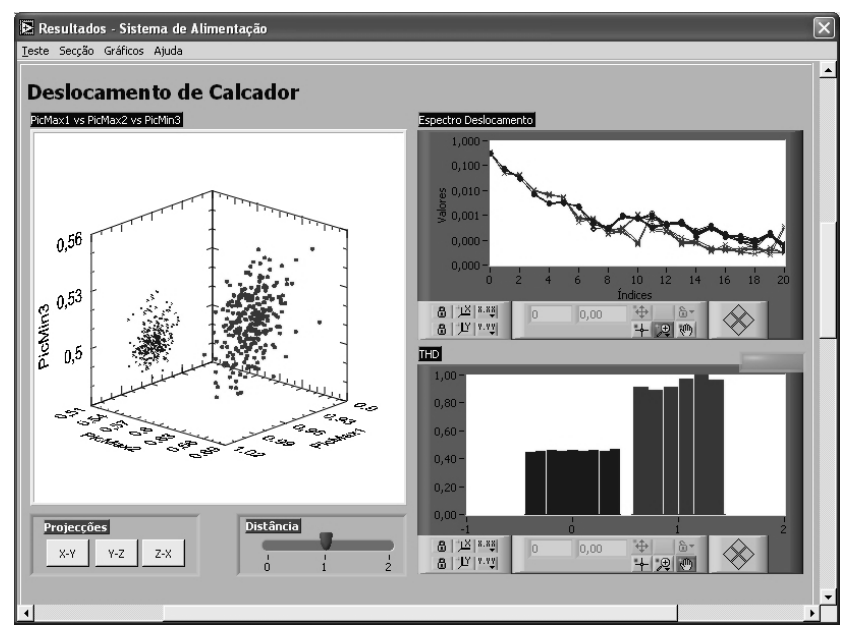

Fig.5: Screenshot of the software, in the example showing data about feeding performance (presser-foot displacement) at 1000 and $3500 \mathrm{spm}$ 


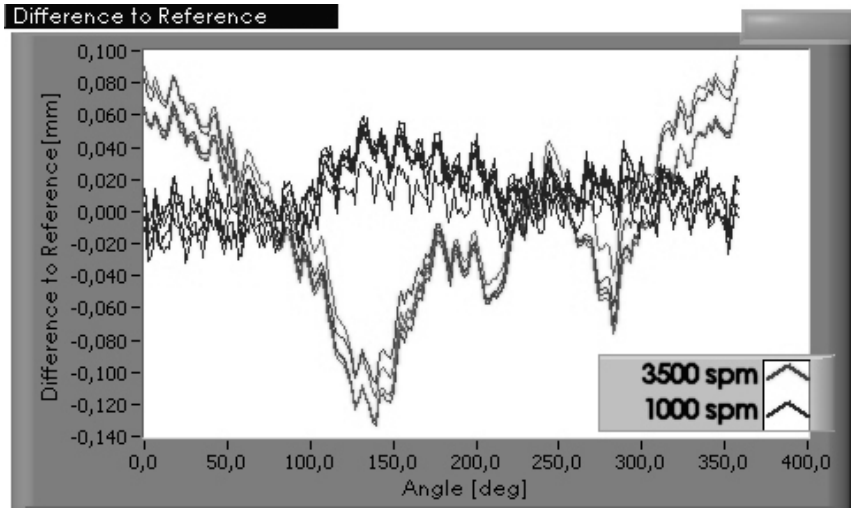

Fig.6: Difference between reference cycle and average displacement signals obtained during the production of seams at 1000 and $3500 \mathrm{spm}$

The presser-foot reference cycle provides a valuable measurement of fabric thickness (under the pressure applied by the presser-foot). It also reveals to some extent the behaviour of the fabrics under compression. This knowledge may be important for the characterisation of fabrics, namely to tune control systems or to describe the fabric sewability ${ }^{I}$. Combined with the active presser foot actuation from Silva [6], more complex test procedures for fabric compressibility are conceivable.

Some useful information is also provided by parameters describing the signals' spectral content. Some harmonic distortion measures clearly indicate varying sewing conditions, but their interpretation must be complemented with other data.

Presser-foot force measurement confirms the observations that are made with the displacement signals. The observation of the force signals may provide some additional insight for research work. However, the parameters provided by the automatic processing tools have not been found to add new monitoring information.

\subsection{Stitch formation}

As far as the experiment design allowed, it has been shown that thread consumption measurement and prediction is a very effective tool to evaluate correct stitch formation regarding tension adjustments. Not only stitch balance, but also the overall tensioning of the seam can be assessed by comparing predicted and measured values.

Fig.7 shows the needle thread tension consumption for three different tension adjustments. The transparent bars represent the theoretical consumption prediction and the dashed lines show the tolerances defined by the user. After analysis of the sewn samples, it has been found that the theoretical prediction is in fact a quite accurate reference for a well-adjusted stitch.

\footnotetext{
${ }^{1}$ Sewability: The ability of a material to be sewn without quality problems
}

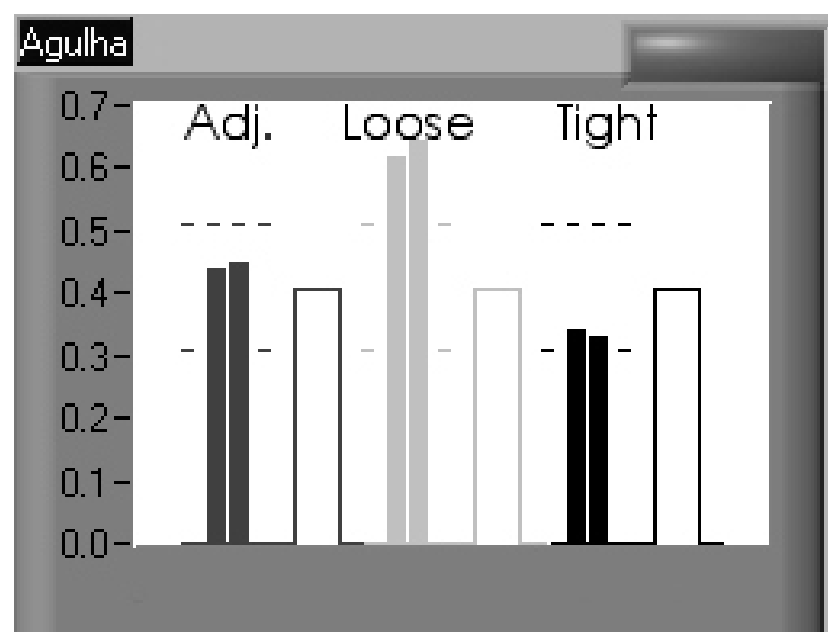

Fig.7: Needle thread consumption per stitch [cm]. Two seams for each situation (adjusted, loose and tight tension). Transparent bars show theoretical prediction of thread consumption

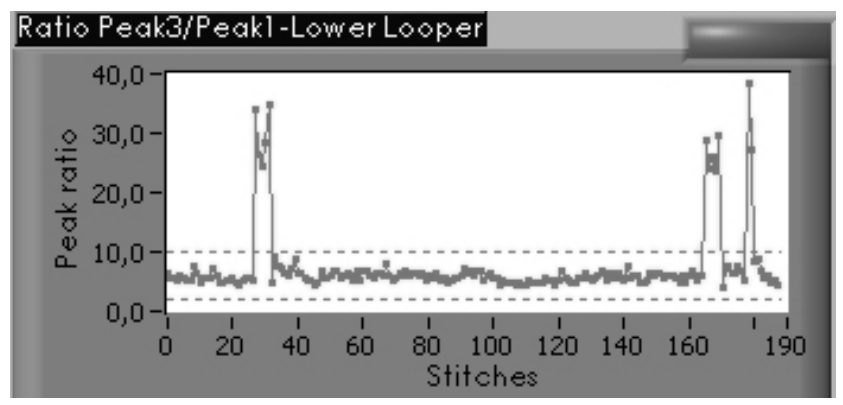

Fig.8: Behaviour of peak ration 3/1 in seams presenting skipped stitches

For the detection of localised defects, parameters obtained from the thread tension signals are an alternative. Some thread tension peaks clearly show the occurrence of skipped stitches ${ }^{2}$ and other, less evident faults. Safer detection margins for these faults are obtained with certain peak ratios (Fig.8). Moreover, some correlation between tension adjustments and these ratios has been observed.

\subsection{Needle penetration}

The forces related to needle penetration forces are the most demanding variables to measure. Great care has been put in the development of tools to perform this task as accurately as possible. Nevertheless, this measurement is partially affected by other forces and thus the measurement is used as an estimation of the needle penetration force.

The problems result mainly from the filtering process that does not eliminate the motional forces completely. In an actual industrial situation, in which the machine is used with thread, thread forces add another effect in the signal.

Still, the results obtained with this system are good and allow the assessment of several important items. It is possible to detect different needle sizes and draw the relation of penetration forces to needle size for a particular

\footnotetext{
2 A skipped stitch occurs if the interlacing of one or several threads or thread loops fails. It is a common defect occurring in practice, and results in a weak spot on the seam
} 
fabric, in a comparative analysis (Fig.9). This relation is different from fabric to fabric and can be linked to its sewability.

The same comparative analysis can be made to compare differently finished fabrics. It has also been possible to distinguish damaged from new needles,. The spread of the measurement may impair the detection of fine differences.

The main factor influencing the penetration value indicated by the system has been found to be sewing speed (Fig.9). A comparative analysis between signals at different speeds is difficult, considering that the same needle produces very different values when speed changes. The increasing value of measured penetration forces with speed is partially due to the higher impact energy between needle and fabric, but it results mainly from the residual mechanical noise still present after the filtering process.

A particular situation occurring during the experiment drew attention to the detection of localised faults. Specifically, with the dyed fabric and using the machine without thread at $3500 \mathrm{spm}$, a clear defect situation occurred due to excessive needle heating. Unfortunately, it was not possible to detect this progressive needle heating in any of the measurements.

The results obtained show that the measurement of needle penetration forces in the current configuration is difficult to apply in an industrial application, in which sewing speed varies in most operations.

A significant improvement to this measurement would be obtained if the force sensor would be placed in a different configuration in order to reduce the motional forces applied to the sensor. This configuration will be tried in future work.

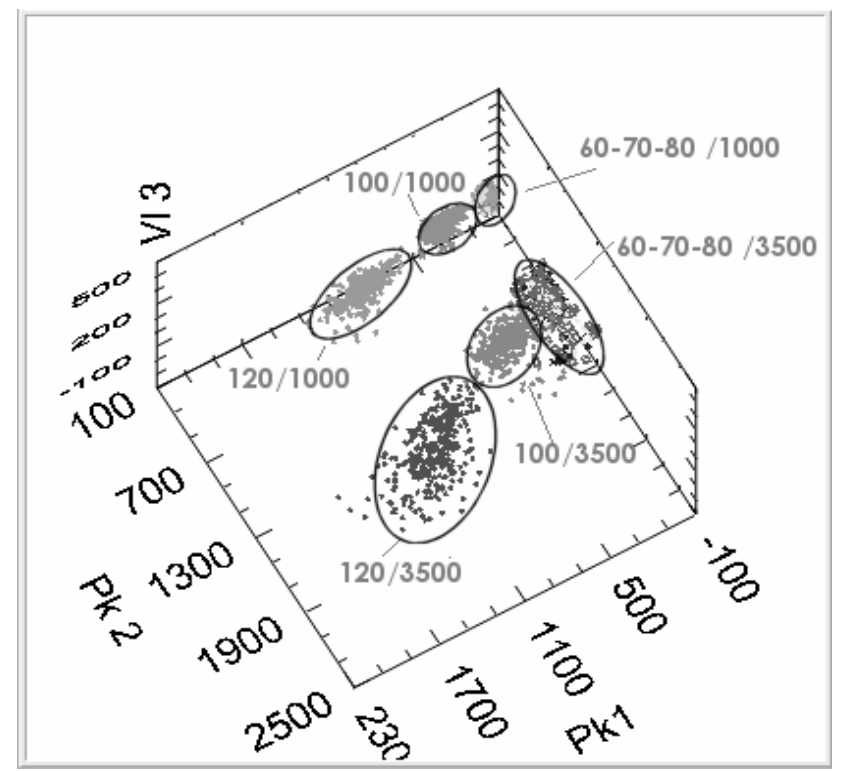

Fig.9: Peak penetration (peak1 and peak2) and withdrawal forces (valley of signal), for $60,70,80,100$ and $120 \mathrm{Nm}$ sized needles, at 1000 and 3500 spm. (Nm: diameter of needle x100)
It is important to emphasize that the measurement showed to be useful for comparative analysis allowing the definition of reference values, definition of optimum sewing conditions for a specific fabric (needle, thread, and speed). This system is useful in industry, where the definition of these optimum sewing conditions prior to production significantly reduces time-to-market and increases the quality of the final product.

\section{Applications and future work}

Currently, very few commercial products (like the Pfaff Doku-Seam) are known to provide some process monitoring capabilities. It is used in airbag manufacturing and monitors thread tension during certain critical seams. Additionally, it monitors the backtack ${ }^{3}$ lever on the machine to assure that the seam never contains a backtack. The system is based on a PC and is able to supply all quantitative data about the seams produced.

But the development of systems for process control is not necessarily limited to high-end machines as the Pfaff Doku-System. The experience and results collected in this project suggest that much simpler devices are conceivable. It is possible to conceive devices devoted to monitoring/controlling a single variable. If developed with the premise of modularity, machines may be upgraded with optional sensors and acquisition systems according to the end user's needs. Modules may be connected and integrated into a higher-level information structure, in which they receive control data according to the product, and provide process data to a central quality management system.

The most immediate applications are the monitoring of thread tensions, material feeding and needle penetration. A monitoring system should consist of a sensor and hardware with conditioning, acquisition and signal processing capabilities. A simple keyboard would serve as input for reference values and tolerances. If the system was complemented with communication capabilities, automatic exchange of process data would be possible.

Another option would be the control of these sewing variables.

In the case of thread tensions, the simplest solution is the use of tensioner compression force sensors with simple indicators, which would allow the quantitative adjustment of thread tension. A completely equipped sewing test rig would be used in sample making as an off-line process planning tool to determine the optimal values for thread tension that can be determined by using, for example, predicted thread consumption. The operator would then quickly set the machine to these values.

\footnotetext{
${ }^{3}$ A backtack is when sewing direction is reversed for a couple of stitches to reinforce the seam at a specific point. In side airbags, where the seam is supposed to break when the airbag is inflated, a backtack, incorrect tension or other kind of defect may impair the function of the airbag
} 
As a next step, it is devisable to replace the traditional tensioner by an active device, using stepper motors to increase spring tension, or a controllable electromagnetic actuator. This would allow an automatic adjustment of tensions. Combined with dynamic thread tension sensors, the process can be monitored and controlled centrally or locally. New possibilities for adaptive control open with this solution, like in-process variation of thread tensions (favourable when different material thicknesses are crossed in one seam, for example).

Feeding system control can use several implementations. The first is an open-loop control in which linear motors vary presser-foot compression force with speed ("Speed responsive Presser-Foot", a technology developed by Pfaff). Silva proposed the replacement of the traditional spring-hinged system by a proportional force solenoid [6].In both cases, if force is to be varied with speed, the exact dependency between both is dependent on the sewn material, and can be determined by the off-line process planning tool. The next stage is a closed-loop control, in which force is adjusted according to the ADL's or reference cycles of the presser-foot.

Te sewing test rig should serve both as a process planning tool, to determine monitoring and control parameters, as well as a sewability tester, to test materials before production. Many enhancements to this sewing test rig are devisable to streamline the definition of control parameter values; in the current state, it is configured as a research tool.

The exact physical implementation of the control devices depends on functional and especially economical factors. In low-end machines, low-cost sensors and hardware have to be developed. However, in high-end machines in which the cost of the acquisition system is low when compared to the cost of the machine, the current configuration is appropriate at least for monitoring applications; it may not be able to keep up with the timing restrictions of a closedloop controller.

\section{Acknowledgements}

The authors are grateful to FCT (Fundação para a Ciência e Tecnologia), project funding POSI/SRI/38944/2001.

\section{References}

[1] Matthews, B. N., Little, T. J., "Sewing Dynamics, Part I: Measuring Sewing Machine Forces at High Speeds", Textile Research Journal, 58, July, 1988, pp. 383-391.

[2] Ferreira,F., A Study of Thread Tensions on a Lockstitch Sewing Machine, $P h D$ thesis, University of Leeds, UK.1991
[3] Chmielowiek, R., Lloyd, D. W., The Measurement of Dynamic Effects in Commercial Sewing Machines, Proceedings of The 3rd Asian Textile Conference, Vol. II, pp. 814-828, 1995

[4] A.M. Rocha, Contribuição para o Controlo Automático de Parâmetros de Costura: Estudo da Penetração da Agulha e Dinâmica da Alimentação, PhD thesis, University of Minho, Portugal. 1996

[5] H.Carvalho, P.Ferreira, A.M.Rocha, J.Monteiro, Signal Feature Extraction for Sewing Analysis using Non-Linear Techniques, Proceedings of the 2003 IEEE International Symposium on Industrial Electronics (ISIE), IEEE, ISBN 0-7803-7912-8, Rio de Janeiro, Brasil, 9-11 Junho 2003

[6] Silva, L. F., Estudo de Mecanismos Alternativos de Controlo do Sistema de Alimentação de Máquinas de Costura Industriais, $P h D$ thesis, University of Minho, Portugal. 2002

[7] Carvalho, Miguel, Estudo das relações entre os parâmetros de controlo, propriedades dos materiais e condições de regulação numa máquina de costura corta-e-cose PhD thesis, University of Minho, Portugal. 2002

[8] H. Carvalho, A. Rocha, J.L. Monteiro, Measurement and Processing of Sewing Variables towards Real-Time Control and Off-line Process Planning, Proceedings of the International Conference on Information Processing and Management of Uncertainty in Knowledge-Based Systems, ISBN 2-9516453-5-X (CD-ROM), Annecy, France, 1-5 July 2002 